\title{
Fast Sensors for Time-of-Flight Imaging Applications
}

\author{
Claire Vallance ${ }^{1}$, Mark Brouard ${ }^{2}$, Alexandra Lauer ${ }^{1}$, Craig Slater $^{1}$, Edward Halford ${ }^{1}$, Benjamin \\ Winter ${ }^{1}$, Simon J. King ${ }^{1}$, Jason W. L. Lee ${ }^{1}$, Daniel Pooley ${ }^{3}$, lain Sedgwick ${ }^{3}$, Renato Turchetta ${ }^{3}$, Andrei \\ Nomerotski ${ }^{4}$, Jaya John John ${ }^{5}$, and Laura Hill ${ }^{5}$
}

\begin{abstract}
${ }^{1}$ Department of Chemistry, University of Oxford, Chemistry Research Laboratory, 12 Mansfield Rd, Oxford OX1 3TA; ${ }^{2}$ Department of Chemistry, University of Oxford, Physical and Theoretical Chemistry Laboratory, South Parks Road, OXford OX1 3QZ; ${ }^{3}$ STFC Rutherford Appleton Laboratory, Harwell, OXfordshire OX11 0QX; ${ }^{4}$ Brookhaven National Laboratory, P.O. Box 5000, Upton, NY 11973-5000, US ; ${ }^{5}$ Department of Physics, University of Oxford, Denys Wilkinson Building, Keble Rd, Oxford OX1 3RH, UK
\end{abstract}

\begin{abstract}
The development of sensors capable of detecting particles and radiation with both high time and high positional resolution is key to improving our understanding in many areas of science. Example applications of such sensors range from fundamental scattering studies of chemical reaction mechanisms through to imaging mass spectrometry of surfaces, neutron scattering studies aimed at probing the structure of materials, and time-resolved fluorescence measurements to elucidate the structure and function of biomolecules. In addition to improved throughput resulting from parallelisation of data collection - imaging of multiple different fragments in velocity-map imaging studies, for example - fast image sensors also offer a number of fundamentally new capabilities in areas such as coincidence detection. In this Perspective, we review recent developments in fast image sensor technology, provide examples of their implementation in a range of different experimental contexts, and discuss potential future developments and applications.
\end{abstract}

\section{Introduction}

Charged particle imaging is widely employed in many areas of science, including particle physics, electron microscopy, ultracold plasma dynamics, chemical photodissociation and reaction dynamics, and imaging mass spectrometry. Imaging is often carried out in tandem with a time-of-flight measurement, which allows particles to be separated according to their kinetic energy, velocity, or mass. As the name suggests, a time-of-flight measurement records the distribution of flight times over a fixed distance for a population of particles released from a source at a well-defined time. Under field-free conditions, the flight time provides a measure of the particle velocity, and therefore its kinetic energy, and provides a means for measuring velocity or kinetic energy distributions for the population of particles. Alternatively, the particles may be accelerated through an electrostatic potential, $V$, to a kinetic energy given by $K=\underline{q V}=1 / 2 m v^{2}$, where $q, m$ and $v$ are the particle's charge, mass, and velocity, respectively. Particles of different mass are therefore accelerated to different velocities, and for a given acceleration potential, each mass has a characteristic arrival time at the detector. Such a measurement forms the basis of time-of-flight mass spectrometry, a ubiquitous technique in the analytical sciences for identifying and quantifying chemical species in a wide variety of contexts. 


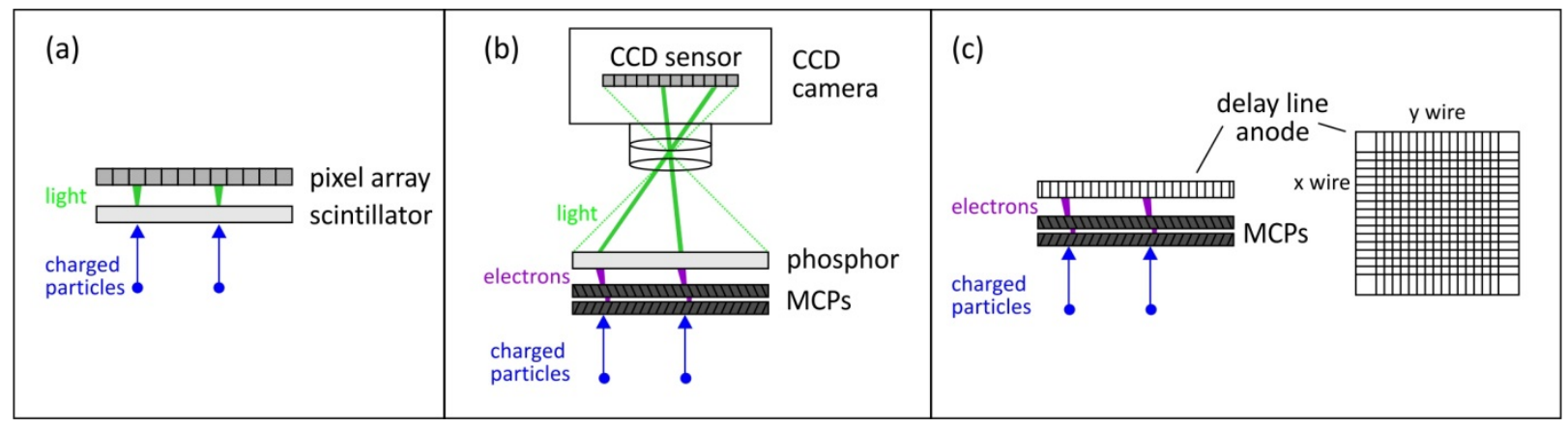

Figure 1: Particle detection schemes: (a) High-energy charged particles can be detected using a scintillator in combination with a pixel or photomultiplier array; (b) Low energy particles can be imaged at high count rates using one or more microchannel plates in combination with a phosphor screen and a camera, and at low count rates using (c) one or more microchannel plates in combination with a delay-line anode.

The detection scheme employed in a particle imaging experiment depends upon the particle energy. Three generic approaches are illustrated schematically in Figure 1. High energy particles, with energies in the range of hundreds of keV or higher, are generally imaged by employing a scintillator screen to convert the charged particle image into an optical image, which is then captured by a photomultiplier or silicon pixel array (Figure 1(a)). It is also common to use pixelated silicon detectors directly, as the incident particles deposit enough energy into the silicon to be registered. At lower incident particle energies, scintillators become inefficient, and alternative detection schemes are required. Typically, one or more microchannel plates are used to convert the incident charged particle into a burst of electrons, which is then incident on a phosphor screen (Figure 1(b)) or delay line anode (Figure 1(c)). In the former case, the optical image on the phosphor screen is recorded using a CCD (charge-coupled device) or CMOS (complementary metal-oxide semiconductor) camera, while in the latter case the position of each charged particle impact is determined by recording the time taken for the resulting current pulse to reach either end of a pair of wires coiled along the $x$ and $y$ axes of the anode.

While the detection schemes outlined above are extremely sensitive, with the ability to detect individual charged particles in most cases, they are limited in their timing and throughput capabilities. A delay line measurement takes around $100 \mathrm{~ns}$, including the time required to reset the electronics for the next measurement. Most delay lines can therefore locate one particle in position and time every 100 ns, with some of the more advanced detectors ${ }^{1}$ incorporating additional anodes to enable them to reduce the measurement dead time to around $10 \mathrm{~ns}$ or better. Detection of several individual particles with flight times separated by more than the measurement time is therefore possible. However, the ability to detect just one (or a few) particles at a time across the whole detector means that in experiments employing delay lines, signal levels must be kept very low, typically to less than one event per cycle, and repetition rates in the $\mathrm{kHz}$ range or higher are required in order to allow data acquisition to proceed at a reasonable rate. Phosphor-based detectors are capable of detecting large numbers of particles simultaneously, but phosphors that are sufficiently bright for use in such detectors tend to have relatively long emission lifetimes of around $100 \mathrm{~ns}$ or more, limiting the achievable time (and therefore mass or energy) resolution. More importantly, the frame rates of most CCD or CMOS cameras used to capture images from the phosphor only permit acquisition of one image per time-of-flight cycle. In an ion imaging experiment, this is not a limitation if there is only one ion of interest to be imaged, as the microchannel plates or an image intensifier positioned in front of the $\mathrm{CCD} / \mathrm{CMOS}$ sensor can be time-gated to the arrival time of the ion of 
interest. However, in experiments for which there is a desire to record multiple images on each experimental cycle, this must generally be achieved by repeating the image acquisition sequence with a number of different time-gates, which quickly becomes a time-consuming process.

Recently, there have been a number of advances in CCD and CMOS image sensor technology which promise to deliver a new generation of detectors combining the best features of each type of detector described above. Such detectors are capable of recording the positions and arrival times of large numbers of particles on every time-of-flight cycle. In this Perspective we will outline the technology underlying these new detectors, and provide an overview of the exciting new science which they are already enabling.

\section{Image Sensor Technology}

The CCD, or charge-coupled device, was first developed in 1969 by Boyle and Smith ${ }^{2}$ at AT\&T Bell Labs. The device was designed to transfer charge in a controlled way along the surface of a semiconductor between a series of storage capacitors, and formed part of a larger project aimed at developing shift registers for new types of memory device. Though Boyle and Smith were concerned purely with developing memory devices, the first publication describing the device ${ }^{2}$ also listed potential applications as a delay line and as an imaging device. The first experimentally realised CCD imaging device was described by Tomsett and coworkers ${ }^{3}$ in 1971.

CCD image sensors are essentially very simple devices for storing and transferring charge. The principle of operation is shown schematically in Figure 2. The device consists of an array of pixels, with one column of pixels constituting the output register. During an exposure, light incident on a pixel generates electron-hole pairs within the silicon, and the charge is stored by a capacitor within the pixel (Figure 2(a)). Charge is moved around within and between pixels by switching the potentials on a series of gating electrodes positioned at the pixel surface (Figure 2(b)). The 'image' is simply a two-dimensional plot of the amount of charge detected within each pixel during an exposure. To read out the sensor at the end of the exposure (as shown in Figure 2(c)), charge is transferred along the rows of pixels such that the entire image is stepped one pixel to the right, placing the charge from the final column of pixels into the output register. The charge is then transferred pixel by pixel along the output register, through a charge amplifier to convert the charge to a voltage, to external readout electronics. The process is repeated for each column of pixels until the entire image has been read out from the camera to the external readout.

The readout process for CCD sensors is inherently slow, and is one of the major limitations of this technology. Standard frame rates are generally on the order of a few tens of frames per second, which is far too slow to allow images to be captured for multiple different flight times on a timescale of tens of microseconds in a time-of-flight experiment. Fast framing CCD cameras provide a potential approach to overcoming this limitation by recording multiple images on-chip before readout. In a framing CCD, each pixel comprises a light-sensitive element, which acquires charge on illumination as described above, together with a set of memory elements. Figure 2(d) shows a schematic of a few pixels from such an array containing 16 elements per pixel. During the first exposure, charge is accumulated at the light-sensitive element. The sensor is then 'clocked' (triggered), and the accumulated charge is moved from the lightsensitive element to the first memory element, leaving the light-sensitive element empty to acquire charge during the second exposure. Following the second exposure, charge from the first filled memory element is moved to the second, and charge from the light-sensitive element is moved to the (now empty) first memory element, and so on until all memory elements are full. At this point the sensor must be read out before further images can be acquired. Charge can be moved between memory elements within a pixel on the nanosecond timescale, allowing very high frame rates to be achieved until all the memory elements within each pixel are filled. The length of each exposure is determined by the clock signals driving the 
frame transfer process. In principle (and in practice for some cameras, for example the commerciallyavailable Dalsa Zenith), these can be programmed independently, which in the context of time-of-flight measurements means that the exposures can be timed to coincide with the arrival times of particles of interest ${ }^{13}$.

(a) Charge collection within a pixel
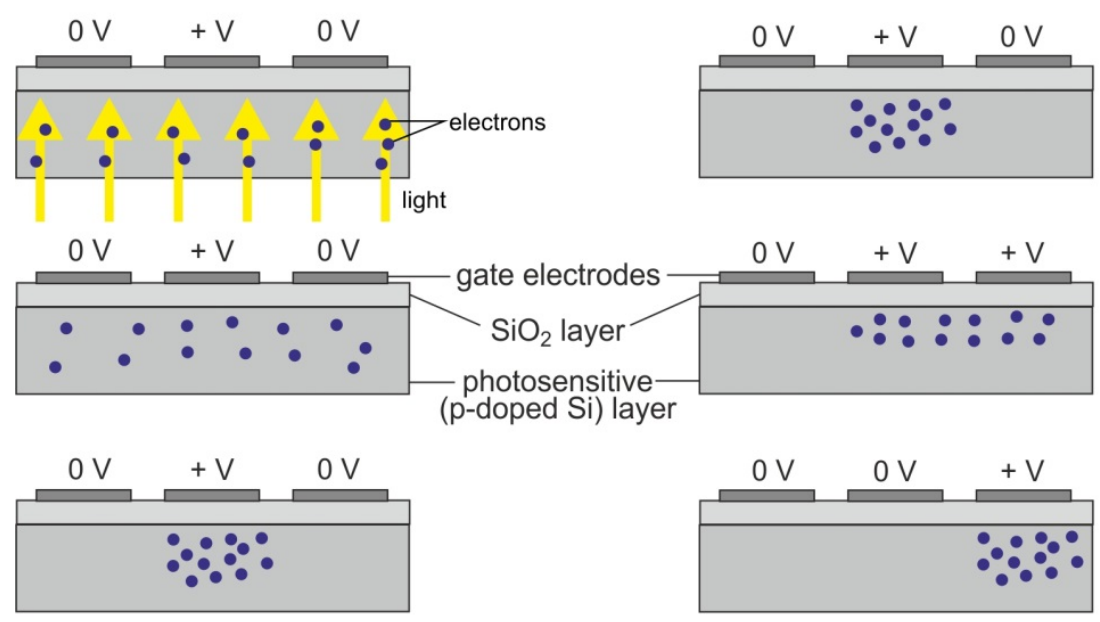

(c) Sensor readout

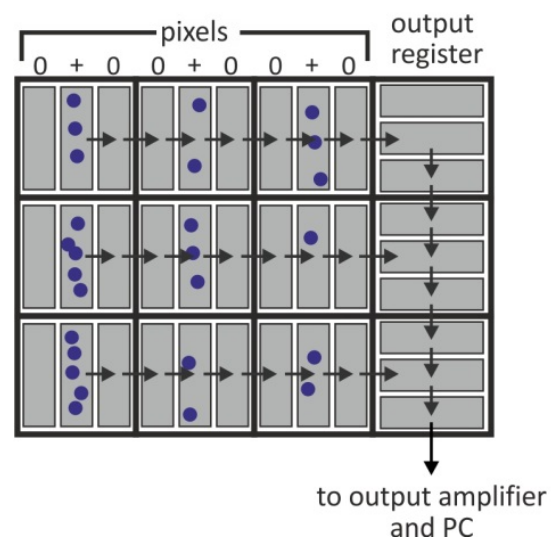

(b) Charge transfer within a pixel
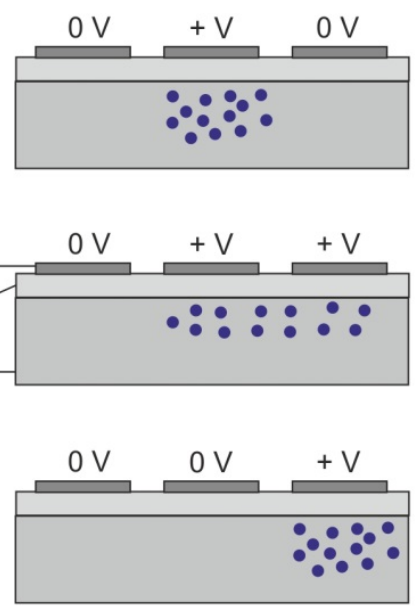

(d) Frame-transfer CCD

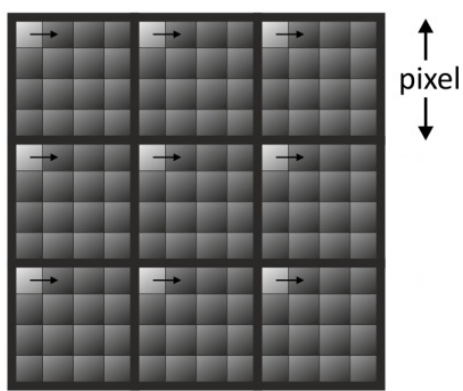

light sensitive element memory register

Figure 2: Schematic illustrating the operation of a simple CCD device (see text for details): (a) charge collection within a pixel; (b) charge transfer within a pixel; (c) sensor readout; (d) a frame-transfer CCD, in which each pixel contains a light sensitive element and an array of memory registers, allowing multiple images to be stored on-chip before sensor readout.

While fast framing CCD cameras are in principle very attractive for time-of-flight imaging applications, there are drawbacks. Frame capture needs to be synchronised with particle arrival times, which means that the time-of-flight spectrum must be known in advance. Also, a limited number of frames can be captured on each time-of-flight cycle, determined by the number of memory registers in each pixel. While this number can be relatively large - some sensors developed by Etoh and coworkers have upwards of 100 registers, for example ${ }^{4}$ - increasing the number of memory elements comes at the expense of sensitivity, since a smaller region of each pixel is photoactive. While this can be mitigated to some extent by the use of microlens arrays to focus incident light onto the sensitive region of each pixel, there is a finite limit to the number of registers that can physically be packed into a pixel. Finally, a data set comprising up 
to or in excess of 100 full-frame images may constitute many gigabytes of data, such that data handling can become a major problem.

Recently developed 'intelligent' CMOS or 'active pixel' sensors provide an alternative approach to time-resolved imaging. In contrast to CCD pixels, which merely acquire and transfer charge, each pixel in an active pixel sensor contains both a photodetector and an active amplifier. The pixels are fabricated using the same CMOS technology employed in microprocessors, static RAM, and other digital control circuits, and share their properties of high noise immunity and low power consumption. Of most interest for high-speed imaging applications, the high density of transistors that can be incorporated into CMOS circuitry means that logic functions can be built into each pixel, opening the way for intelligent pixel sensors. Two such sensors have recently come to the attention of the time-of-flight imaging community: the TimePix/MediPix sensor originally developed at CERN for particle physics applications ${ }^{5}$; and the PImMS (Pixel Imaging Mass Spectrometry) sensor developed in Oxford, originally for applications in imaging mass spectrometry ${ }^{6}$.

Though the fabrication processes and detailed specifications of the TimePix and PImMS sensors differ, the basic operating principles are similar. Rather than acquiring complete image frames, as in the case of the CCD sensors described above, each pixel contains one or more counters, and operates independently to detect particles. In the case of the $256 \times 256$ pixel TimePix sensor, the single counter within each pixel can be set (independently for each pixel in the array) to operate in one of three modes. On each acquisition cycle the counter either: (i) counts the number of incident particles; (ii) records the arrival time of a single incident particle; or (iii) uses the number of clock cycles for which the signal remains above a pre-defined threshold to determine the particle energy. Mode (ii) is of most interest for time-offlight imaging applications, allowing particle arrival times to be recorded with around $10 \mathrm{~ns}$ timing resolution. Rather than detecting light, the TimePix sensor is configured for direct detection of charged particles. The surface of each pixel comprises a metal pad which transfers charge directly to the pixel. If signal levels are high enough then the TimePix sensor can be bump-bonded to a separate silicon pixel array and used as a stand-alone particle detector via generation of charge within the silicon on particle impact. Alternatively, if signal amplification is required then the sensor can be used in combination with one or more microchannel plates ${ }^{7}$. In the latter case, electrons emitted from the back face of the microchannel plate are collected directly by the metal pads of the TimePix sensor, and the silicon sensor array is not required.

In contrast, the PImMS sensor is configured solely to record particle arrival times. The sensor can store up to four arrival times per pixel to allow detection of multiple particles on each time-of-flight cycle, with a best time resolution similar to that of the TimePix sensor, at $12.5 \mathrm{~ns}$. At the end of an acquisition cycle, the sensor reads out an array of 'time stamps', which the acquisition software converts into a list of $(x, y, t)$ data points for each detected particle. The capacity to store four arrival times within each pixel mitigates against a reduction in detection sensitivity for heavier or slower particles, which would not be detected if a pixel had already detected an earlier particle on a given time-of-flight cycle. The prototype 72 x 72 pixel PImMS1 sensor is now in increasingly widespread use, and a larger 324 x 324 pixel version is currently in the testing stages. While the sensor can detect high energy charged particles directly (see Section 3.4 for an example), it is configured primarily as a visible light sensor, and is most commonly used in combination with an $\mathrm{MCP} /$ phosphor position-sensitive detector. Future backthinned versions of the sensor will be capable of detecting low energy charged particles in vacuum.

Intelligent pixel sensors largely overcome the shortcomings of framing CCD image sensors alluded to earlier. Since each pixel logs particle arrival times independently, no prior information is needed on the time-of-flight spectrum; in fact, the time-of-flight spectrum may readily be extracted from the recorded data set simply by integrating over the spatial coordinates. The mass resolution is determined by the time resolution of the sensor, which in turn is determined by design parameters, such as the base frequency of 
the pixel clock, and also fundamental physical limitations, such as the time required for charge generated within the sensor to diffuse to the detection diodes within each pixel. For the PImMS sensor, this ultimate limit is around $5 \mathrm{~ns}$. While the number of different arrival times that may be imaged by a CCD sensor is limited by the number of memory registers in each pixel, in an intelligent CMOS sensor it is limited only by the bit depth of the pixel counters, which at 12 bits (4096) for the PImmS sensor and 14 bits (16384) for the TimePix sensor, is unlikely to be a limiting factor. Finally, instead of recording large numbers of complete frames, both the PImMS and TimePix sensors store and read out only the counter values within each pixel, vastly improving efficiency and data handling relative to framing cameras through a dramatic reduction in the amount of recorded information.

While we have focused our discussion on two ultrafast CMOS image sensors, there are a number of others currently in development. For example, the Gigatracker hybrid pixel detector ${ }^{8}$, currently being developed for use on the NA62 Experiment at CERN, will record particle arrival times with better than 200 ps resolution, though the array size is relatively small. As we shall see in Section 4 , there is also a great deal of current research into new pixel technologies with the potential to provide imaging capabilities on the sub-nanosecond timescale, with single-photon detection sensitivity. However, before looking to the future, we will first provide an overview of some of the areas in which fast time-of-flight imaging sensors are beginning to make an impact.

\section{Applications}

\subsection{Reaction dynamics (velocity-map imaging)}

Velocity-map imaging ${ }^{9.10}$ (VMI) is now well established in the field of small-molecule reaction dynamics as the method of choice for studying molecular photofragmentation events and other process. Following a chemical reaction or photofragmentation, the product velocity distributions are highly sensitive to the detailed dynamics of the process under study, and when combined with state-of-the-art theory, can provide information on the potential energy surface or surfaces accessed during the process, together with bond strengths, transition state geometries, and energy partitioning between translational and internal modes of motion. While early VMI studies focused on elucidating the fragmentation dynamics of diatomic and triatomic molecules in as much detail as possible, in recent years the focus has shifted towards larger chemical species of relevance in biology, synthetic organic chemistry, and other areas of interest. VMI is now routinely used to study the fragmentation dynamics of a variety of organic molecules, and even relatively large chemical species have been observed to yield structured, information-rich images. The very high density of states in larger molecules means that achieving individual quantum state resolution is of less interest than in small-molecule studies, and imaging studies are often concerned more with obtaining a general picture of energy partitioning, as well as investigating the competition between different fragmentation pathways. When studying a diatomic or triatomic molecule, with a single accessible photofragmentation pathway, one can often obtain a fairly complete picture of the fragmentation dynamics through imaging of a single fragment in combination with basic principles of energy and momentum conservation. However, for larger molecules, with multiple fragmentation pathways, it becomes extremely desirable to image multiple fragmentation products on each time-of-flight cycle.

Previous efforts to achieve multimass velocity-map imaging have relied on separating the various masses spatially on the detector by incorporation of an additional electric or magnetic field along the ion flight path ${ }^{11,12}$. The accessible mass range in a given measurement is then severely limited by the physical size of the detector relative to that of the individual images. Delay-line detectors also provide capabilities for multimass detection, as explained in Section 1, but only at extremely low data rates such that only one 
or a few ions arrives within a given $\sim 100$ ns measurement time. These problems are overcome by ultrafast image sensors, since the images for individual masses can now be resolved in time rather than in space, and a large number of individual pixels (essentially parallel delay lines) are available for particle detection.

While the field is still in its infancy, there have already been a number of demonstrations in which ultrafast image sensors have been used for multimass imaging and other chemical dynamics experiments. In the first example, Brouard et $a l^{13}$ employed a fast framing CCD camera capable of acquiring 16 frames on-chip to image the various fragments formed following UV photolysis of $\mathrm{CS}_{2}$ and $\mathrm{CH}_{3} \mathrm{~S}_{2} \mathrm{CH}_{3}$. Though the framing camera had limited resolution due to its small $64 \times 64$ pixel array, the images - some of which are shown in Figure 3(a) - and the corresponding speed and angular distributions determined for the various fragments were in good agreement with those acquired fragment by fragment using a conventional CCD camera, providing solid proof-of-principle for the approach. While ideally suited to the relatively small molecules imaged in this study, which had only a small number of fragmentation channels, for larger molecules the number of memory registers within each pixel would be insufficient to record images for every fragment, particularly since a number of the available frames must be used as 'spacer' frames to fill in time gaps between mass peaks of interest. These problems have largely been overcome by the 'eventtriggered' architecture of the TimePix and PImMS sensors.

The TimePix sensor has been used for velocity-map imaging of both electrons and ions, and has the unique feature amongst VMI detectors that it can be positioned inside the vacuum chamber to detect charged particles directly. The first reported experiments, by Gademann et $a l^{14}$, imaged photoelectrons from the $355 \mathrm{~nm}$ ionization of Xe. Electrons were imaged directly by the TimePix sensor, removing the high vacuum requirements imposed by detectors employing microchannel plates. While MCPs cannot be operated at pressures higher than around $10^{-5} \mathrm{mbar}$ owing to the risk of catastrophic damage caused by electrical arcing, the TimePix sensor can in principle be used at atmospheric pressure and higher. In the study on Xe photoionization, the authors were able to record images at pressures up to $10^{-4} \mathrm{mbar}$, with loss of signal at higher pressures due to scattering of the molecular beam and/or photoelectrons. Activation of a pixel requires the generation of several hundred electrons within the silicon layer of the sensor, which is easily achieved when the electrons produced in the photoionization process of interest are accelerated to energies of around $5 \mathrm{kV}$ or higher. In this case, the TimePix chip can be used as a standalone sensor. At lower signal levels, the sensor can be used together with one or more MCPs to provide amplification.

While providing a first proof-of-principle of the TimePix detector as a detector for VMI measurements, the photoelectron imaging experiments did not exploit the potential for time-resolved multimass detection. However, this was demonstrated shortly afterwards through ion imaging experiments reported by Jungmann et al $^{7}$, and also in previously unpublished work carried out in our own laboratory ${ }^{15}$ at around the same time. In both sets of experiments, the TimePix sensor was positioned inside the vacuum behind a pair of chevron-mounted MCPs, replacing the phosphor screen of a conventional imaging detector. A potential of a few hundred volts was applied in order to accelerate electrons from the back face of the MCPs towards the sensor, and in the two experiments the setup was used to image $\mathrm{NO}^{+}$ions formed in the $451.64 \mathrm{~nm}$ photolysis of $\mathrm{NO}_{2}$, and $\mathrm{CS}^{+}$and $\mathrm{CS}_{2}{ }^{+}$ions formed in the 193 $\mathrm{nm}$ dissociative ionization of $\mathrm{CS}_{2}$, respectively. Figure $3(\mathrm{~b})$ shows an image of $\mathrm{CS}^{+}$ions recorded using the TimePix sensor in our laboratory. Jungmann et al were also able to demonstrate slice imaging in their experiments, an application which will be considered in more detail later.

The PImMS sensor has also been employed as a detector in time-resolved velocity-map imaging studies into gas-phase ${ }^{16}$ and surface ${ }^{17}$ photochemistry. Configured as an optical sensor, the camera is simply mounted outside the vacuum in place of the CCD camera in a conventional VMI detection setup. 
Figures 3(c) and 3(d) show example data sets for electrons and ions formed in the dissociative ionization of $\mathrm{Br}_{2}$ at $446.32 \mathrm{~nm}$, and ions formed in the dissociative ionization of $\mathrm{N}, \mathrm{N}$-dimethylformamide at $193 \mathrm{~nm}$, respectively. In the $\mathrm{Br}_{2}$ experiments, the polarity of the velocity-mapping electrodes was switched following extraction of the electrons in order to allow both the electrons and the ions to be imaged on each time-of-flight cycle. As noted earlier, the four timing registers in each pixel ensure that the pixels have sufficient capacity to detect heavier masses when one or more lighter ions have already been detected, precluding a bias towards lighter ions in the detection step. In the $\mathrm{Br}_{2}$ experiments, the two isotopes of atomic bromine are clearly resolved. The experiments on $\mathrm{N}, \mathrm{N}$-dimethylformamide yield a large number of different fragment ions, some of which result from clustering of the parent molecule within the molecular beam. Signal from the parent ion was extremely intense, and was therefore time-gated out of the signal to avoid damage to the microchannel plates. The large number of different fragments would make conventional mass-by-mass imaging extremely time consuming relative to the present experiments, in which images were acquired simultaneously for all masses. It is clear from Figure 3(d) that each fragment has a characteristic velocity distribution, ranging from extremely narrow velocity distributions for parent ions, for which the velocity distribution simply reflects that of the molecules within the molecular beam, to distributions extending to very high velocities for ions formed in high-energy fragmentation processes. A comprehensive analysis of this data is still underway, but is expected to provide interesting insights into the photofragmentation dynamics of the $\mathrm{N}, \mathrm{N}$-dimethylformamide molecule, which provides a simple model for the peptide bond.

(a)

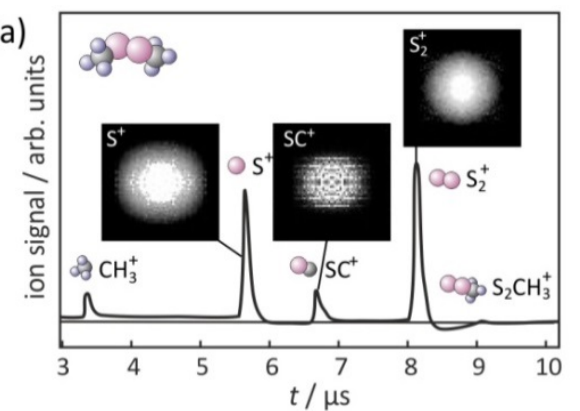

(c)

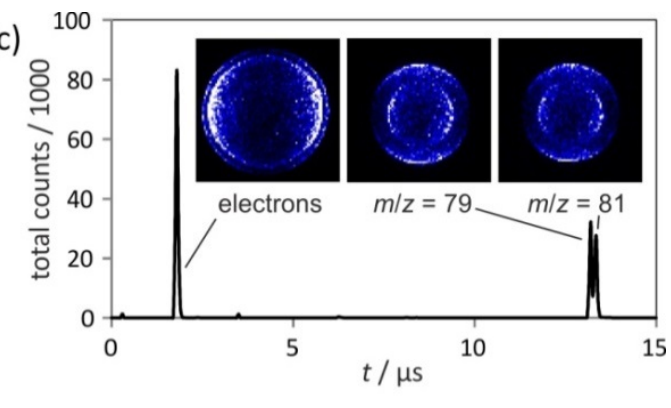

(b)

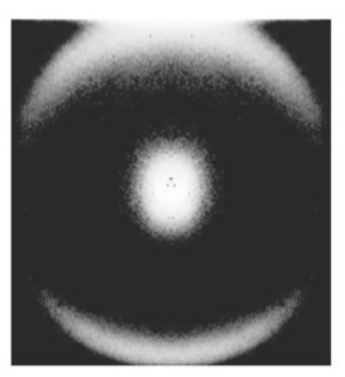

(d)

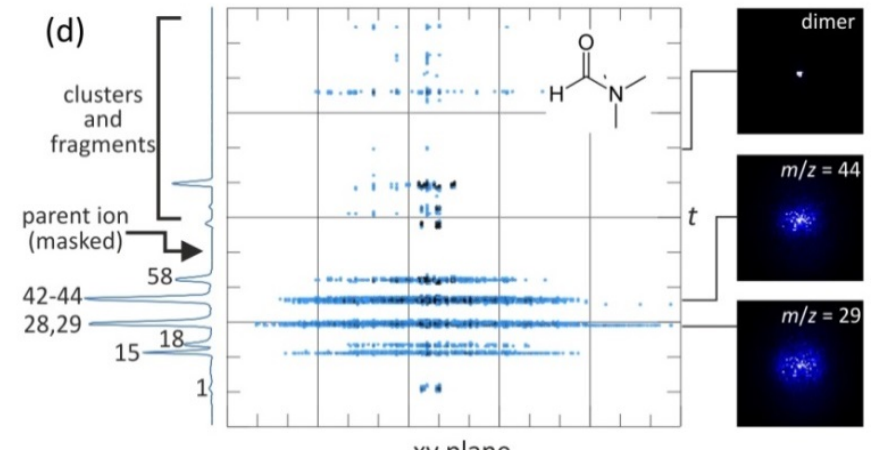

xy plane

Figure 3: Multimass velocity-map imaging: (a) Images of fragments formed in the $193 \mathrm{~nm}$ photolysis of dimethyldisulphide, recorded with the Dalsa Zenith fast-framing CCD camera ${ }^{13}$; (b) Image of $\mathrm{CS}^{+}$ions formed in the 193 ns dissociative ionization of $\mathrm{CS}_{2}$, recorded using the TimePix sensor; (c) Electrons and ions formed in the $446.32 \mathrm{~nm}$ dissociative ionization of $\mathrm{Br}_{2}$, recorded using the PImMS sensor; (d) Complete data set for $193 \mathrm{~nm}$ photolysis of $\mathrm{N}, \mathrm{N}$ dimethylformamide, with selected images for the dimer and peptide bond fission products. (c) and (d) adapted from reference 16.

We note that while multimass VMI has so far been limited mainly to studies of photofragmentation dynamics, there is considerable potential for exploiting the technique in mass spectrometric fragmentation 
studies. Biological molecules in the gas phase are increasingly being studied via tandem mass spectrometry or 'MS/MS' techniques, in which the molecule of interest is selected by a first mass spectrometry stage, before being fragmented by one of a variety of collisional, laser-induced, or electron-induced processes. The fragment ions are then analysed in a second mass spectrometry stage. The ability to image the velocity distributions of the fragment ions formed in such a process has the potential to provide a significant increase in the information content of data from such studies. In addition to yielding insight into the energetics of the fragmentation process, such methods could be used to probe bond lengths and internal excitation, as well as providing a rapid means of distinguishing between parent and daughter ions via analysis of the kinetic energy release distributions of the ions.

Focusing again on chemical dynamics, ultrafast imaging detectors provide exciting opportunities for 3D imaging of molecular fragmentation processes, as shown in Figure 4. In most VMI experiments, the measured image is a two-dimensional projection of the complete three-dimensional product velocity distribution, and one of a variety of mathematical transforms must be employed in order to reconstruct the full 3D distribution. A variant of the technique is 'slice imaging', in which the imaging optics are designed such that the ion cloud is expanded along the ToF axis rather than being compressed into a 'pancake' as it reaches the detector ${ }^{18,19,20}$. When a camera with a suitably fast shutter is used (e.g. a time-gateable intensified CCD camera), or by pulsing the detector on for only a very brief period of time, it becomes possible to image a single slice through the centre of the distribution. In many cases, the ion distribution is cylindrically symmetric, and this single slice contains all of the information necessary for reconstructing the full 3D velocity distribution. However, this is not always the case. The approach described above can also be used to image non-cylindrically-symmetric distributions, but in this case the 3D distribution must be built up slice-by-slice over a large number of acquisition cycles by stepping the time gate through the full width of the ion arrival time distribution. This process is both time-consuming and susceptible to the effects of experimental drift over the timescales required to record a complete data set. This can be an important consideration when attempting to detect subtle features in the velocity distribution caused by atomic or molecular alignment or orientation, for example ${ }^{21}$. Using a 'single slice' approach also precludes the measurement of correlations between fragments, a technique we will discuss in some detail in Section 3.2.

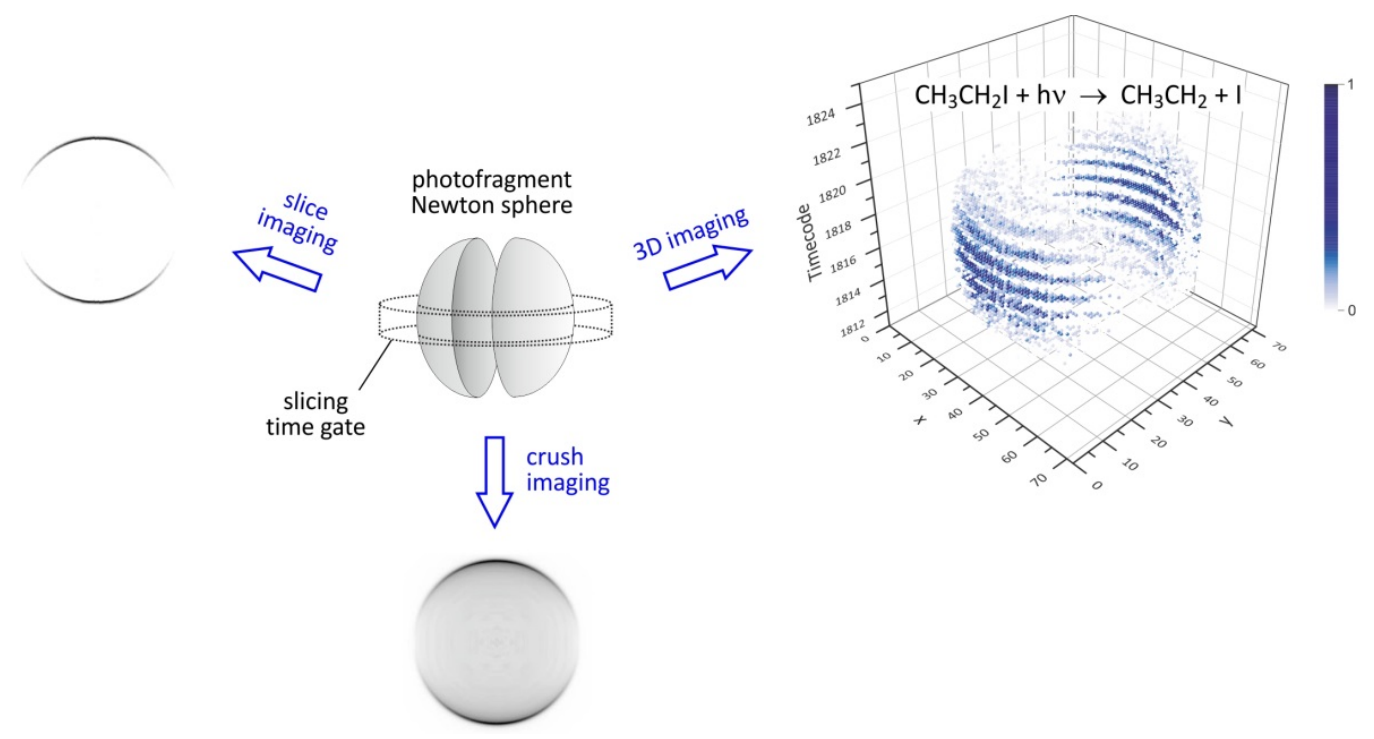

Figure 4: 3D imaging: (a) standard 'crush' imaging; (b) slice imaging; (c) 3D imaging; (d) 3D images of $\mathrm{I}^{+}$ions produced in the $245 \mathrm{~nm}$ photolysis of iodoethane, recording using the PImMS1 sensor $^{22}$. 
An ultrafast imaging detector is capable of recording the full 3D scattering distribution directly, eliminating the need for mathematical reconstruction, and providing a much more general and powerful approach than existing methods. In addition to recording the 3D distribution, if individual slices through the distribution are desired, these may be generated simply by selecting a subset of events recorded within a user-specified time window from the full 3D data set. This approach was first demonstrated by Jungmann et al in their VMI study of $\mathrm{NO}_{2}$ photofragmentation using the TimePix sensor ${ }^{7}$. The time resolution of the sensor was reported to be $12.5 \mathrm{~ns}$, which is broadly similar to that achievable when gating the camera or detector in conventional 'single slice' imaging. The authors were able to show that the velocity resolution achieved in the 3D data set was superior to that obtained when the data was integrated over the time axis to produce a standard 'crushed' image, which was then analysed using standard Abel inversion methods.

Recent experiments ${ }^{22}$ have demonstrated that it is also possible to achieve similar results using the PImMS sensor coupled to an MCP/phosphor ion detector. The plot on the right hand side of Figure 4 shows a 3D representation of the entire Newton sphere of the $\mathrm{I}^{+}$ions produced following photodissociation of iodoethane at $245 \mathrm{~nm}$, as recorded using the PImMS1 camera. The camera clock cycle was $12.5 \mathrm{~ns}$, the same as that reported for the Timepix sensor in the work of Jungmann et al. ${ }^{7}$ Each slice is well resolved, with the comparatively long 100 ns decay lifetime of the P47 phosphor having a relatively small effect on the time resolution. This is due to the fact that the pixels in the image sensor trigger on the rising edge of a signal from the phosphor.

\subsection{Coincidence measurements}

Coincidence detection techniques ${ }^{23}$, which probe correlations between individual fragments produced from the same parent molecule, provide a powerful tool in studying the photofragmentation or photoinduced reaction dynamics of gas-phase molecules. The fragments probed may either be two ions, in which case the experiment is referred to as a photoion-photoion coincidence (PIPICO) measurement ${ }^{24,25}$, or an ion and the partner electron, known as a photoelectron-photoion coincidence (PEPICO) experiment ${ }^{26}$. Recording the correlation between two particles arising from the same parent molecule clearly requires both particles to be detected within the same time-of-flight cycle. In the case of PIPICO imaging measurements, this precludes the use of a conventional CCD camera as part of the detection system, as the readout speed of such cameras is too slow to acquire more than one image on each experimental cycle. In the case of PEPICO, the electrons and ions are usually accelerated in opposite directions and recorded using two separate detection systems. A pair of conventional CCD cameras can therefore be used, with each camera time-gated to the arrival time of the particle of interest. Janssen et $\mathrm{al}^{27}$ and Brouard et $\mathrm{al}^{16}$ have recently demonstrated that by rapid switching of the polarity of the ion optics used to extract the photofragmentation products to the detector, it is in fact possible to record images of both electrons and positive ions on a single detector. The ion optics are initially tuned to image the electrons, and then switched in polarity to image the ions as soon as the electrons have cleared the extraction region. The short delay in extracting the ions, during which the electric field has the 'wrong' polarity, induces a negligible disturbance to the ion trajectories. When employing this single-detector version of PEPICO, the detector requirements are identical to those for PIPICO, i.e. position and time (mass) resolved detection is required on the timescale of the ion or electron arrival times.

Coincidence measurements are normally carried out under experimental conditions tuned such that the number of fragmentation events is kept to less than one event per acquisition or time-of-flight cycle. This ensures that any two (or more) particles detected on a given cycle must have originated from the same parent molecule. The detector typically consists of one or more MCPs coupled to a delay-line 
anode. To maximise the data acquisition rate, experiments are generally carried out at kilohertz repetition rates, but even so, it often takes many days or even weeks to acquire a data set of sufficiently high quality.

An alternative approach to coincidence detection has recently been demonstrated by Brouard, Stapelfeldt and coworkers ${ }^{28,29}$ in which the requirement for ultra-low count rates on each acquisition cycle is removed. Instead, the PImMS sensor is used to record position and timing information for each detected ion at relatively high count rates, such that ions are detected from multiple parent fragmentation events on each time-of-flight cycle, and the correlation information is extracted from the data via statistical covariance analysis $^{30,31}$. The approach provides the same information as obtained in the more traditional approaches to coincidence detection, but the acquisition time is shortened considerably, and the method is also compatible with typical repetition rates of the nanosecond pulsed laser systems used in many reaction dynamics experiments.

Several sets of experiments have been carried out to demonstrate the new approach. Electrons and bromine atoms formed in the dissociative UV ionization of $\mathrm{Br}_{2}$ have been imaged in a single-detector high -count-rate PEPICO measurement ${ }^{16}$, with separate images recorded for the two isotopes of atomic bromine. In another PEPICO measurement by Brouard, Whitaker, et $\mathrm{al}^{32}$, electrons and various fragment ions arising from the dissociative ionization of $\mathrm{NO}_{2}$ have been imaged. It was shown that distinct photoelectron images can be extracted for electrons correlated with different fragment ions. These experiments also demonstrate that correlation techniques are not necessarily limited to identifying coincidences between only two particles, and that muti-electron multi-ion correlation is also likely to be possible.
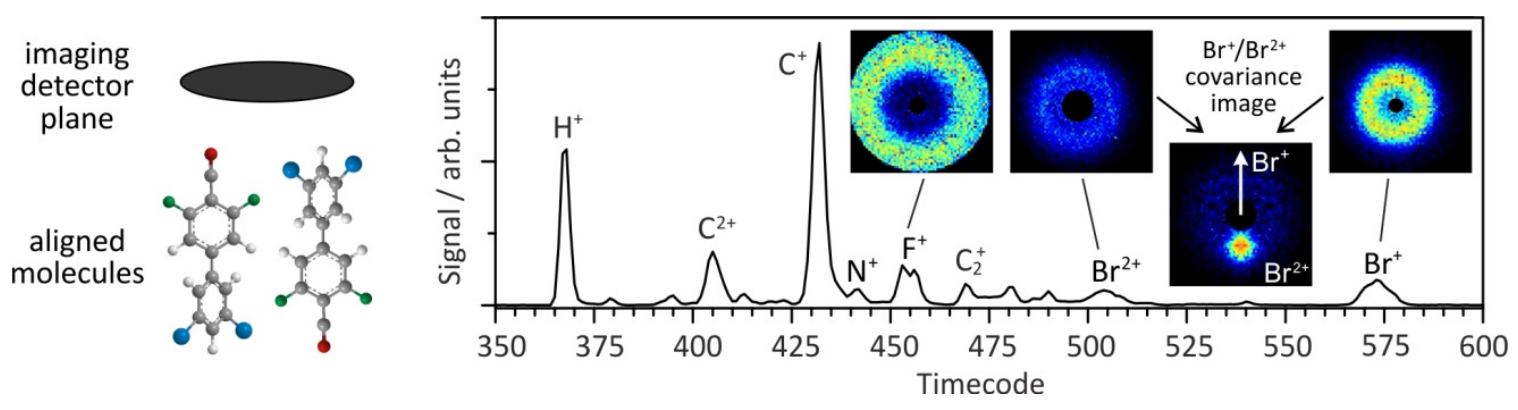

Figure 5: Coulomb explosion coincidence imaging of a biphenyl derivative ${ }^{28}$. The molecules are laser-aligned as shown relative to the imaging detector plane prior to Coulomb explosion $(C=$ grey, $\mathrm{H}=$ white $\mathrm{N}=$ red, $\mathrm{Br}=$ blue, $\mathrm{F}=$ green). Following the Coulomb explosion, the PImMS sensor records velocity-map images for each fragment in tandem with the ToF mass spectrum. Selected images are shown for the $\mathrm{F}^{+}, \mathrm{Br}^{2+}$, and $\mathrm{Br}^{+}$fragments. Covariance images reveal correlations between the velocities of pairs of fragments, illustrated here with a covariance image of $\mathrm{Br}^{+}$and $\mathrm{Br}^{2+}$ fragments, which recoil at 180 degrees from each other when viewed from the detector.

The method has also been applied to PIPICO measurements. In recent experiments by Brouard, Stapelfeldt, and coworkers ${ }^{28}$, a biphenyl derivative was spatially aligned using a nanosecond laser pulse, and then Coulomb-exploded using an intense femtosecond laser pulse. The correlated velocity distributions of the various atomic fragments formed in the Coulomb explosion, measured by velocity-map imaging with the PImMS sensor, reflect the molecular structure when the explosion was initiated. The 
resulting covariance images therefore reveal a great deal of structural information; in the experiments on the biphenyl species, for example, the covariance images clearly revealed the dihedral angle between the two phenyl rings ${ }^{28}$, as well as the relative angles between a number of other chemical bonds within the structure. Figure 5 provides an illustrative example, in which a covariance image of two atomic bromine fragments resulting from the Coulomb explosion clearly shows them recoiling in opposite directions as viewed along the ToF axis. In the future, by combining coincidence methods with 3D imaging, it may prove possible to extract such information without the requirement for pre-aligning the molecule under study prior to the Coulomb explosion.

\subsection{Surface analysis (spatial-map imaging)}

Mass spectrometric techniques have found a vast array of applications, spanning areas as diverse as the identification and structural analysis of proteins, peptides and oligonucleotides; drug discovery, pharmacokinetics; breath gas monitoring; quality control; reaction kinetics measurements; quantitation of complex chemical mixtures; and geochemical and archaeological dating. Imaging techniques add a new dimension to mass spectrometry, and are attracting considerable interest within the analytical sciences. Imaging mass spectrometry of a surface yields a type of 'chemical microscopy', allowing the visualisation of analytes ranging from trace metals to peptides, proteins and surface lipids directly from the surface of a biological or other sample, without the need for fluorescent or other labelling.

To date, most approaches to imaging mass spectrometry involve rastering either an ion beam or a laser beam across the surface - usually achieved by mounting the sample on an $x y$ stage - and recording an array of conventional (usually time-of-flight) mass spectra of the ions produced for each set of $(x, y)$ coordinates of the beam. Images of the spatial distribution for any analyte may then be reconstructed from the $(x, y)$ dependence of the intensity of the corresponding mass peak. This is known as the 'microprobe' approach to imaging mass spectrometry. The spatial resolution in this approach is determined primarily by the spot size of the ion or laser beam, and ranges from tens of nanometres for some ion beam methods to a few tens of microns (down to around a micron for the most highly optimised instruments $^{33}$ ) for most laser methods ${ }^{34}$. A variety of ion generation methods are available, with the optimum method for a given application depending on the nature of the sample under study. Matrixassisted laser desorption ionization (MALDI) offers a 'soft' ionization method leading to little molecular fragmentation, and has been used to image distributions of intact lipids, peptides and proteins up to a mass of around 100,000 Da in a wide variety of tissue samples ${ }^{35}$. Alternatively, ionization may be achieved via secondary ion emission on bombarding the surface with a focused ion beam. While the ion beams traditionally used in secondary ion mass spectrometry (SIMS) led to virtually complete molecular fragmentation, such that the method was best suited to elemental rather than molecular surface analysis, ion sources have now been developed that permit molecular imaging, and the extremely small spot size that can be achieved with a focused ion beam have allowed sub cellular imaging of smaller organic compounds $(<1000 \mathrm{Da})$ to be achieved ${ }^{36}$. Atmospheric-pressure ionization methods such as desorption electrospray ionization (DESI) have also been applied in imaging mass spectrometry. DESI has the advantage that no prior sample preparation is required, and that the sample does not need to be introduced into the vacuum system of the mass spectrometer. However, the spatial resolution achievable is limited ${ }^{36}$, at around $200-400 \mu \mathrm{m}$.

Recently, there has been considerable interest in an alternative, 'microscope' mode of imaging ${ }^{39}$, in which a large area of the sample (several $\mathrm{mm}^{2}$ ) is illuminated by a diffuse laser or ion beam, and the ion 
optics system employed is designed such that a stigmatic image of the complete sample is recorded on a position-sensitive detector. The microprobe and microscope approaches have recently been reviewed in the context of their suitability for biomolecular and biomedical imaging ${ }^{37}$. Microscope-mode imaging has the potential to reduce the data acquisition time from many hours - as is currently the case for microprobe tissue imaging experiments - to a few minutes. However, until recently the ability to acquire images for a range of masses on this timescale has been beyond the reach of existing image sensors. This is set to change with the advent of intelligent active pixel sensors of the type described in Section 2. The ability of sensors such as PImMS and TimePix to acquire images for many masses simultaneously allows the spatiallyresolved chemical composition of the sample to be determined on each time-of-flight cycle ${ }^{38}$. In the following, we review progress to date on exploiting the capabilities of intelligent pixel sensors in imaging mass spectrometry.

One of the first modern microscope-mode imaging mass spectrometers was constructed by Heeren and coworkers ${ }^{39,40}$, who modified a commercial TRIFT II SIMS instrument. The spectrometer can be used either with a laser-based ionization method, such as MALDI, or with an ion beam for SIMS experiments. Klerk et al. demonstrated the imaging capabilities of the instrument by imaging sample arrays of biologically relevant substances, such as cholesterol and angiotensin II, acquiring data mass by mass with a conventional CCD camera ${ }^{41}$. Multi-mass imaging was first demonstrated following replacement of the original standard MCP/phosphor screen/CCD detection system with a hexanode delay line detector ${ }^{42,43}$. A sample consisting of a hexagonal TEM grid coated in a photosensitive dye was bombarded with ions from a $\mathrm{C}_{60}$ primary ion beam, defocused to a spot diameter of $250 \mu \mathrm{m}$. The resulting ion images, recorded for masses up to $m / z \approx 500$, were determined to have a spatial resolution of $4 \mu \mathrm{m}$. Though these experiments allowed the collection of ion images with high time and lateral resolution, the limitation on the number of events that can be recorded in each ToF cycle, as imposed by the delay line detector, has restricted further applications.

The first intelligent pixel sensor applied to imaging mass spectrometry was the 'quad' version of the Medipix 2 sensor $^{44}$, a precursor to TimePix, in which four $256 \times 256$ pixel sensor chips are arranged in a $2 \times 2$ array to give a total of $512 \times 512$ pixels. The sensor was positioned to detect the electrons from the back face of the microchannel plate ion detector. Because the MediPix sensor was not able to record timing information, a selective ion blanker was used to gate the detection to a single mass for each image acquisition. In operation on the TRIFT instrument described above, spatial images were recorded with a lateral resolution of $6 \mu \mathrm{m}$ employing SIMS to generate ions from the sample surface, and 8-10 $\mu \mathrm{m}$ when employing laser desorption ionization methods. In later work, the MediPix sensor was replaced with a TimePix sensor, which allowed images to be recorded simultaneously for multiple masses. The instrument performance was demonstrated by imaging a sample consisting of peptide mixtures arrayed in a grid pattern ${ }^{45}$. The time-of-flight spectrum recorded by the TimePix detector exhibited a lower time resolution but a higher signal-to-noise ratio than that obtained using the standard approach of recording signals directly from the MCP via a time-to-digital or analogue-to-digital converter. The spectrometer has since been used in a number of further proof-of-concept demonstrations of its capabilities. Bio- and macromolecules have been imaged both in isolation and in mouse testis tissue samples ${ }^{46,47}$ (see Figure 6(a)); spectra and images have been recorded in both positive and negative ion modes ${ }^{48}$; and by postaccelerating the extracted ions, the mass range has been extended up to $100 \mathrm{kDa}$ while at the same time improving the spectral quality. 
(a)

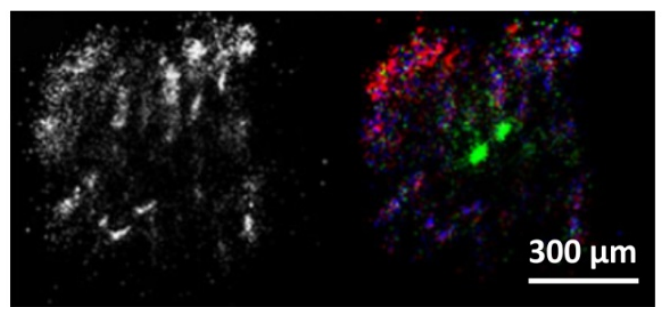

(b)

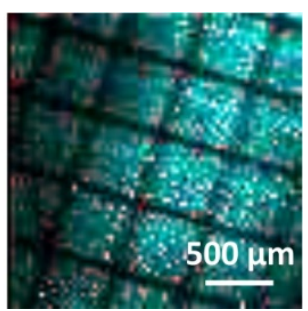

Figure 6: (a) Imaging mass spectrometry with the TimePix sensor. The sample is a thin section through a mouse testis. The image on the left shows the total ion image, while the image on the right shows mass selected images $(\mathrm{m} / \mathrm{z}=491-495$ in blue, $\mathrm{m} / \mathrm{z}=595-599$ in green, $\mathrm{m} / \mathrm{z}=$ 624 in red) at a spatial resolution of $740 \mathrm{~nm}$ per pixel. Adapted from Jungmann et al. ${ }^{46}$; (b) Imaging mass spectrometry with the PImMS1 sensor ${ }^{52}$ : the sample is Exalite 398 dye electrosprayed through a Ni mesh stencil (67 um wire diameter, 500 um wire spacing) at a spatial resolution of $31 \mu \mathrm{m}$, determined by the $2 \mathrm{~mm}$ field of view and $72 \times 72$ pixel sensor array size.

A very promising design of microscope-mode imaging mass spectrometer is the MULTUM II multiturn time-of-flight imaging instrument developed by Toyoda and coworkers ${ }^{49}$. The instrument achieves high time-of-flight resolution by injecting the ions into a closed loop formed from four toroidal electric sectors, which circulate the ions multiple times in a 'bow-tie' shaped orbit order to achieve a long flight path, before ejecting them towards the detector. Increasing the number of ion circulations improves the time-of-flight resolution, and therefore the mass resolution, but at the cost of spatial distortions. For 500 cycles, a mass resolution of 130000 was achieved, but at the cost of distorted images. Switching to a single-pass imaging mode yielded a spatial resolution of $4 \mu \mathrm{m}$ over a $400 \mu \mathrm{m}$ field of view ${ }^{50}$. To achieve multi-mass imaging, the same researchers have developed a delay line detector for use with their instrument, which they estimate will yield spatial and mass resolutions of $12 \mu \mathrm{m}$ and 15000 , respectively ${ }^{51}$. To our knowledge, multi-turn ToF instruments have not yet been used in combination with intelligent pixel sensors.

More recently, Brouard, Vallance, and coworkers have demonstrated spatial-map imaging of large area (up to $5 \mathrm{~mm}$ ) samples with $20 \mu \mathrm{m}$ resolution using an ion lens based on a velocity-map imaging lens and a detection system employing an $\mathrm{MCP} /$ phosphor ion detector coupled to the PImMS sensor ${ }^{52}$. A test image of ions from a laser dye sprayed onto the sample plate through a nickel mesh stencil is shown in Figure 6(b). The simple linear extraction method employed in these experiments yielded a relatively low mass resolution. However, the mass resolution has recently been improved upon by employing the postextraction differential acceleration (PEDA) method, originally developed by Aoiki et al ${ }^{53}$ and improved by Winter et $\mathrm{al}^{54}$. The method uses a five-electrode ion lens, with the first two electrodes forming the repeller and extractor elements of a standard spatial-map imaging lens, and the following three electrodes forming an Einzel lens. Once the ions have cleared the extractor plate (lens 2), this plate is pulsed to a higher potential, providing an additional 'kick' that time-focuses the initial velocity spread of the extracted ions as they reach the detector. The method may be used to record mass spectra and images for ions of a range of masses around a central 'optimized' mass, which can be tuned to any mass of interest while maintaining the spatial mapping properties ${ }^{54}$. Using a standard CCD camera, a mass resolution of 2200 and spatial resolution of sub $20 \mu \mathrm{m}$ for a field of view of $4 \mathrm{~mm}$ has been achieved, and future experiments employing the PImMS sensor are planned.

While ultrafast image sensors have so far only been demonstrated in a small number of microscope-mode imaging mass spectrometry measurements, the potential for vastly reducing acquisition 
times relative to the widely-employed microprobe mode imaging methods has generated considerable interest amongst instrument manufacturers in commercialising the technology in the medium term.

\subsection{Other applications}

While this Perspective has focused on applications of fast image sensors in the fields of reaction dynamics and imaging mass spectrometry, combining time-of-flight measurements with imaging opens up a vast array of applications in other areas such as fluorescence lifetime imaging, time-resolved electron and x-ray imaging and diffraction studies, high energy particle physics, and neutron science. To highlight the versatility of such detectors, we will outline briefly some recent proof-of-concept work in which the PImMS sensor has been used to perform neutron time-of-flight imaging ${ }^{55}$.

Neutron ToF imaging differs from other applications discussed so far in this Perspective, in that the time-of-flight measurement is now used to determine the kinetic energy of the detected particles, rather than their mass-to-charge ratio. Combining the ToF information with high-resolution imaging provides a powerful non-destructive analysis technique or chemical and materials science, since each pixel now uniquely records the crystallographic structure, texture, and strain through the irradiated region of the sample $^{56}$. Example applications include the mapping of magnetic flux lines in superconductors ${ }^{57}$, and material studies into residual strain in welds ${ }^{58}$. By combining neutron TOF imaging with sample rotation, full four-dimensional tomographic images of the sample can be generated, yielding information that cannot be accessed via any other technique.

In common with most VMI measurements, it has generally only been possible to acquire timeresolved neutron images by stepping the time-gate of an intensified CCD camera ${ }^{59}$ through the neutron arrival time distribution, a time consuming process which represents a highly inefficient use of neutron beam time at spallation sources ${ }^{60}$. Time-resolved imaging sensors, such as the TimePix ${ }^{61,62}$ and PImMS sensors, provide an alternative approach.

Neutron measurements with the PImMS sensor have been performed at the Oxfordshire-based ISIS neutron spallation facility. ISIS generates pulses of neutrons with kinetic energies ranging from $0.1 \mathrm{meV}$ to $800 \mathrm{MeV}$ through proton bombardment of a tungsten target. The silicon-based PImMS sensor is not intrinsically sensitive to neutrons ${ }^{1}$, and is therefore coated with a thin gadolinium conversion material. Neutron capture by gadolinium results in emission of $\sim 70 \mathrm{keV}$ conversion electrons, which are detected directly by the sensor. Additionally, the time bin interval is increased so that the acquisition cycle matches the $\sim 20$ ms width of the neutron ToF distribution. The resulting 'GaDMOS' neutron detector is able to record the position and arrival time of every detected neutron in each pulse, allowing images to be reconstructed over the neutron energies of interest. If a sample is placed in the neutron beam, the ToF distribution recorded by each pixel will in general show a series of 'dips' (Bragg edges) at characteristic energies, due to diffraction of the neutron beam by the sample.

Figure 7 shows some early results from the GaDMOS detector. In Figure 7(a), Bragg edges are clearly visible in the neutron time-of-flight distribution recorded following transmission of the neutron beam through an Fe sample. Figure 7 (b) shows an optical image of a cadmium mask used to test the imaging capabilities of the detector. Low energy neutrons are blocked by the cadmium, so only neutrons passing through the $1 \mathrm{~mm}$ circular apertures in the mask are transmitted to the detector. Figure 7(c) shows the neutron image recorded by the GaDMOS detector at low neutron energies when the mask is in place. It

\footnotetext{
${ }^{1}$ The PImMS sensor in fact does have a very small inherent sensitivity to neutrons due to the small thermal neutron cross section of the ${ }^{10} \mathrm{~B}$ used to dope the silicon.
} 
is clear that the detector simultaneously offers high time and spatial resolution for time-resolved neutron imaging, opening up exciting possibilities in the field of neutron science.

(a)

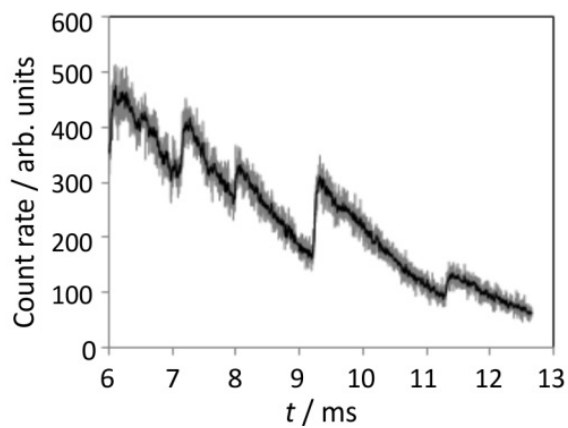

(b)

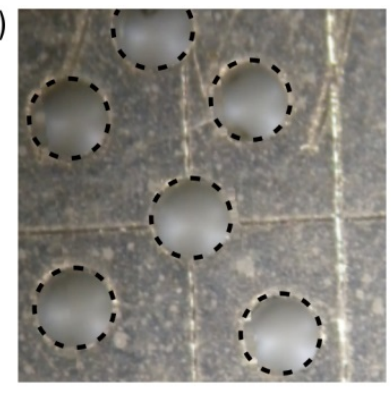

(c)

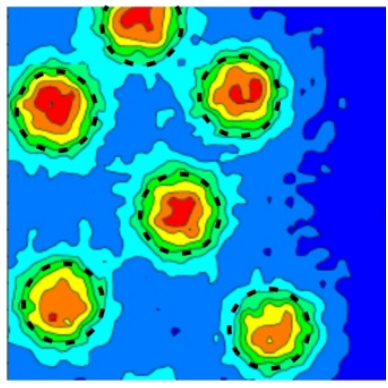

Figure 7: Neutron detection with the GaDMOS detector, which employs a gadolinium-coated PImMS sensor chip ${ }^{55}$ : (a) iron Bragg edges in the neutron transmission spectrum, as recorded by the GaDMOS detector; (b) optical image of the cadmium mask used to block the neutron beam for imaging tests; (c) images of neutrons transmitted through the $1 \mathrm{~mm}$ apertures in the $C d$ mask, as recorded by the GaDMOS detector.

\section{The Future}

While the capabilities of existing sensors such as PImMS and TimePix are already impressive, a number of new technologies are being developed which promise a step change in sensitivity and time resolution for future sensors, opening up the regime of sub-nanosecond, single-photon measurements. We round off this Perspective by considering one of the most promising of these, which is likely to enter mainstream use within the next few years. A number of research groups and companies are working on incorporating single-photon avalanche photodiode (SPAD) technology into pixel array sensors. In a conventional CMOS pixel, the ultimate time resolution is limited by the time required for the charge created by incident photons to migrate to the detection diode. This depends on the thickness of the epitaxial layer and on the electric field within the pixel, but for the PImMS sensor is around 5-10 ns, for example. In contrast, a SPAD works by biasing a pair of diodes in a metastable condition such that a single electron reaching the junction triggers an avalanche mechanism, resulting in a large current flow and a correspondingly large signal, which can be transformed into a digital pulse. The avalanche mechanism is extremely fast, and can be used to provide a timing measurement on the timescale of $<100 \mathrm{ps}$, with single-photon detection sensitivity.

When combined with a fast phosphor or scintillator, the single-photon detection capability of SPAD-based pixels opens up the intriguing possibility of developing a new direct ion detector as an alternative to microchannel-plate-based detectors. As noted in Section 1, scintillators are widely used to detect high-energy ions, usually in combination with a photomultiplier tube, but their efficiency drops off dramatically at low incident energies, with each ion generating only a few photons at ion energies of a few keV. SPAD sensors have sufficient sensitivity to detect even these few photons, and a sensor coated in a thin layer of an appropriate scintillator therefore constitutes a stand-alone ion detector with a time resolution determined by the luminescence lifetime of the scintillator. Ion detectors of this type will be extremely robust relative to MCPs, with the ability to operate at pressures up to atmospheric pressure and higher. This yields intriguing possibilities for new MS techniques operating in a higher pressure regime than traditional methods, subject to the development of suitable ion lens designs or other ion separation technologies. CMOS-based sensors will also be considerably lower in cost than traditional ion detectors, 
since their production benefits from the large investment in mainstream CMOS technology widely used for consumer products. The detectors may also, of course, be used for non-imaging applications, in which the signal is simply integrated over the whole sensor to yield a sensitive large-area ion detector or silicon photomultiplier.

Proof-of-concept for such a sensor has already been provided by Wilman et $a l^{63}$, who used a commercially available multipixel photon counting (MPPC) detector (Hamamatsu) coupled to an LYSO scintillator crystal to record time-of-flight mass spectra of butanone following $193 \mathrm{~nm}$ UV ionization. The MPPC comprises an array of SPADs connected in parallel such that the total output of all of the pixels in the sensor is integrated to yield the signal. The time resolution in these measurements was limited to $49 \mathrm{~ns}$ by the decay lifetime of the scintillator. More recent experiments ${ }^{64}$, in which the LYSO scintillator was replaced by a more efficient fast organic scintillator, have yielded greatly improved time resolution and signal intensity. A sample data set is shown in Figure 8.

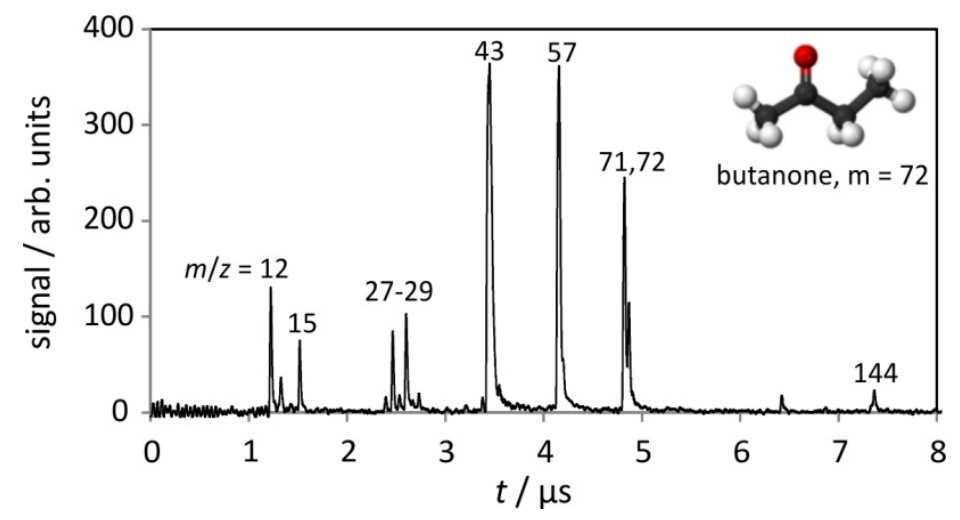

Figure 8: Mass spectrum for butanone recorded using a fast organic scintillator coupled to a commercially-available MPPC detector.

As we have seen, fast image sensors have the potential to revolutionise time-resolved particle imaging experiments, offering drastically reduced data acquisition times in chemical dynamics experiments and imaging mass spectrometry, and opening up entirely new scientific possibilities in areas such as coincidence detection, and new timescales at greatly reduced cost for applications such as time-resolved neutron imaging. While intelligent pixel sensors are a relatively new development, they are already capturing the imagination of the scientific community, and will no doubt be implemented in an ever increasing variety of applications as the technology becomes established and they become more widely available.

\section{Acknowledgements}

We acknowledge funding from the ERC through Starting Independent Researcher Grant 200733 'ImageMS', from the EPSRC Programme Grant EP/G00224X/1, from the STFC via a PNPAS award and mini-IPS grant ST/J002895/1, from the Marie Curie Initial Training Network 238671 'ICONIC', and from the DFG through grant LA 3209/1-1. We would like to thank Henrik Stapelfeldt, Lauge Christensen, Jens Nielsen, and Benjamin Shepperson for allowing us to use the Coulomb explosion data presented in Section 3.2; Ben Whitaker, with whom we have collaborated on some of the reaction dynamics work; and E. Schooneveld, N. Rhodes and R. Farrow for their assistance with the neutron experiments described in Section 3.4 


\section{References}

${ }^{1}$ F. Osterdahl, S. Rosen, V. Bednarska, A. Petrignani, F. Hellberg, M. Larsson, and W. J. van der Zande, J. Phys: Conf. Ser., 2005, 4, 286-289.

${ }^{2}$ W. S. Boyle and G. E. Smith, Bell Sys. Tech. J., 1970, 49(4), 587-593.

${ }^{3}$ G. F. Amelio, M. F. Tompsett, and G. E. Smith, Bell Sys. Tech. J, 1970, 49(4), 593-600.

${ }^{4}$ T. G. Etoh, D. V. T. Son, T. Yamada, and E. Charbon, Sensors, 2013, 13, 4640-4658.

${ }^{5}$ X. Llopart, R. Ballabriga, M. Campbell, L.Tlustos, and W. Wong, Nucl. Instrum. Meth. A, 2007, 581(1-2), $485-494$.

${ }^{6}$ A. Nomerotski, M. Brouard, E. Campbell, A. Clark, J. Crooks, J. Fopma, J. J. John, A. J. Johnsen, C. Slater, R. Turchetta, C. Vallance, E. Wilman and W. H. Yuen, J. Inst., 2010, 5, C07007/0-6.

7 J. H. Jungmann, A. Gijbertsen, J. Visser, J. Visschers, R. M. A. Heeren, and M. J. J. Vrakking, Rev. Sci. Instrum, 2010, 81, $103112-103118$.

${ }^{8}$ M. Fiorini, V. Carassiti, A. Ceccucci, E. Cortina, A. Cotta Ramusino, G. Dellacasa, S. Garbolino, P. Jarron, J. Kaplon, A. Kluge, A. Mapelli, F. Marchetto, E. Martin, S. Maroiu, G. Mazza, M. Morel, M. Nov, G. Nuessle, F. Petrucci, P. Riedler, G. Aglieri Rinella, A. Rivetti, and S. Tiuraniemi, S., Nucl. Instrum. Meth. A, 2011, 628, 292-295.

${ }^{9}$ Chandler, D. W.; Houston, P. L. , J. Chem. Phys. 1987, 87, 1445-1447.

${ }^{10}$ A. T. J. B. Eppink and D. H. Parker, Rev. Sci. Instrum., 1997, 68(9), 3477-3484.

${ }^{11}$ M. H. Kim, B. D. Leskiw, L. Shen, and A. G. Suits, Int. J. Mass Spectrom., 2006, 252, 73-78.

${ }^{12}$ S. T. Tsai, C. K. Lin, Y. T. Lee, and C. K. Ni, Rev. Sci. Instrum., 2001, 72, 1963-1969.

${ }^{13}$ M. Brouard, E. K. Campbell, A. J. Johnsen, C. Vallance, W. H. Yuen, and A. Nomerotski, Rev. Sci. Instrum., 2008, 79, $123115-123122$.

${ }^{14}$ G. Gademann, Y. Huismans, A. Gijsbertsen, J. Jungmann, J. Visschers, and M. J. Vrakking, Rev. Sci. Instrum., 2009, 80, 103105-103111.

${ }^{15}$ E. Wilman, C. Vallance, and A. Nomerotski, unpublished data (2009).

${ }^{16}$ A. T. Clark, J. P. Crooks, I. Sedgwick, R. Turchetta, J. W. L. Lee, J. J. John, E. S. Wilman, L. Hill, E. Halford, C. S. Slater, B. Winter, W. H. Yuen, S. H. Gardiner, M. L. Lipciuc, M. Brouard, A. Nomerotski, and C. Vallance, J. Phys. Chem. A, 2012, 116, 10897-10903.

${ }^{17}$ M. D. Kershis, D. P. Wilson, M. G. White, J. J. John, A. Nomerotski, M. Brouard, J. W. L. Lee, C. Vallance, and R. Turchetta, J. Chem. Phys., submitted July 2013.

${ }^{18}$ C. R. Gebhardt, T. P. Rakitzis, P. C. Samartzis, V. Ladopoulos, and T. N. Kitsopoulos, Rev. Sci. Instrum., 2001, 72, 38483853.

19 J. J. Lin, J. Zhou, W. Shiu, and K. Liu, Rev. Sci. Instrum., 2003, 74, 2495-2500.

${ }^{20}$ D. Townsend, M. P. Minitti, and A. G. Suits, Rev. Sci. Instrum., 2003, 74, 2530-2539.

${ }^{21}$ C. Vallance, Phys. Chem. Chem. Phys., 2011, 13, 14427-14441.

${ }^{22}$ C. Slater, S. Blake, M.Brouard, J.J. John, A. Nomerotski, R. Turchetta, and C. Vallance, manuscript in preparation, 2013.

${ }^{23}$ R. E Continetti, Annu. Rev. Phys. Chem., 2001, 52, 165-192.

${ }^{24}$ G. Dujardin, S. Leach, O. Dutuit, P. M. Guyon, and M. Richard-Viard, Chemical Physics, 1984, 88, 339-353.

${ }^{25}$ D.M. Curtis and J.H.D. Eland, Int. J. of Mass Spectrom. and lon Proc., 1985, 63, 241-264.

${ }^{26}$ T. Baer, Int. J. of Mass Spectrom., 2000, 200, 443-457.

${ }^{27}$ C. Stefan Lehmann, N. Bhargava Ram, and Maurice H. M. Janssen, Rev. Sci. Instrum., 2012, 83, 093103-093106.

${ }^{28}$ C. S. Slater, A. Lauer, S. Blake, M. Brouard, A. Nomerotski, R. Turchetta, C. Vallance, L. Christensen, J. H. Nielsen, B. Shepperson, and H. Stapelfeldt, manuscript in preparation, 2013.

${ }^{29}$ J. Hansen, J. Nielsen, C. Madsen, A. Lindhardt, M. Johansson, T. Skrydstrup, L. Madsen, and H. Stapelfeldt, J. Chem. Phys., 2012, 136, 204310-204319.

${ }^{30}$ C. Cornaggia, J. Phys. B: At. Mol. Opt. Phys., 2012, 45, 085602-085610.

${ }^{31}$ L. J. Frasinski, K. Codling and P. A. Hatherly, Science, 1989, 246, 1029-1031.

${ }^{32}$ C. S. Slater, B. Whitaker, M. Brouard et al.; manuscript in preparation, 2013.

${ }^{33}$ A. Zavalin, E. M. Todd, P. D. Rawhouser, J. Yang, J. L. Norris, R. M. Caprioli, J. Mass Spectrom., 2012, 47, $1473-1481$.

34 J. D. Dorrestein and P. C. Watrous, Nature Reviews: Microbiology, 2011, 9, 683-694.

${ }^{35}$ S. Khatib-Shahidi, M. Andersson, J. L. Herman, T. A. Gillespie, R. M. Caprioli, Anal. Chem., 2006, 78, 6448-6456.

36 S. S. Rubakhin, J. V. Sweedler, Mass Spectrometry Imaging - Principles and Protocols (Humana Press, Springer, 2010).

${ }^{37}$ J. H. Jungmann, R.M.A. Heeren, J. Proteomics, 2012, 75, 5077-5092.

38 J. H. Jungmann and R. M. A. Heeren, Rapid Commun. Mass Spectrom., 2013, 27, 1-23.

${ }^{39}$ S. L. Luxembourg, T. H. Mize, L. A. McDonnell, R. M. A. Heeren, Anal. Chem. 2004, 76, 5339-5344.

${ }^{40}$ B. W. Schueler, Microsc. Microanal. Microstruct. 1992, 3, 119-139. 
${ }^{41}$ L. A. Klerk, A. F. M. Altelaar, M. Froesch, L. A. McDonnell, R. M. A. Heeren, Int. J. Mass Spectrom., 2009, 285, 19-25.

${ }^{42}$ M. Froesch, S.L. Luxembourg, D. Verheijde, R.M. A. Heeren, Eur. J. Mass Spectrom., 2010, 16 (1), 35-45.

${ }^{43}$ L. A. Klerk, N. P. Lockyer, A. Kharchenko, L. MacAleese, P. Y. W. Dankers, J. C. Vickerman, R. M. A. Heeren, Anal. Chem., 2010, 82, 801-807.

${ }^{44}$ J. H. Jungmann, L. MacAleese, R. Buijs, F. Giskes, A. D. Snaijer, J. Visser, J. Visschers, M. J. J. Vrakking, R. M. A. Heeren, J. Am. Soc. Mass. Spectrom., 2010, 21, 2023-2030.

${ }^{45}$ J. H. Jungmann, L. MacAleese, J. Visser, M. J. J. Vrakking, R. M. A. Heeren, Anal. Chem., 2011, 83, 7888-7894.

${ }^{46}$ J. H. Jungmann, D. F. Smith, L. MacAleese, I. Klinkert, J. Visser, R. M. A. Heeren, J. Am. Soc. Mass. Spectrom., 2012, 23, 1679-1688.

${ }^{47}$ A. Kiss, J. H. Jungmann, D. F. Smith, R. M. A. Heeren, Rev. Sci. Instrum., 2013, 84, 013704-013710.

${ }^{48}$ J. H. Jungmann, D. F. Smith, A. Kiss, L. MacAleese, R. Buijs, R. M. A. Heeren, Int. J. Mass Spectrom., 2013, 341-342, 32-44.

${ }^{49}$ H. Hazama, J. Aoki, H. Nagao, R. Suzuki, T. Tashima, K.-i. Fujii, K. Masuda, K. Awazu, M. Toyoda, Y. Naito, Appl. Surf. Sci., 2008, 255, 1257-1263.

${ }^{50}$ H. Hazama, H. Yoshimura, J. Aoki, H. Nagao, M. Toyoda, K. Masuda, K. Fujii, T. Tashima, Y. Naito, K. Awazu, J.Biomed. Opt., 2011, 16, 046007/1-046007/7.

${ }^{51}$ H. Yoshimura, H. Hazama, J. Aoiki, M. Toyoda, Y. Naito, and K. Awazu, Jpn. J. Appl. Phys., 2011, 50, $056701-056707$.

${ }^{52}$ M. Brouard, E. Halford, A. Lauer, C. S. Slater, B. Winter, W. H. Yuen, J. J. John, L. Hill, A. Nomerotski, A. Clark, J. Crooks, I. Sedgwick, R. Turchetta, J. W. L. Lee, C. Vallance, E. Wilman, Rev. Sci. Instrum., 2012, 83, 114101-114109.

${ }^{53}$ J. Aoki, H. Hazama, M. Toyada, J. Mass Spectrom. Soc. Jpn., 2011, 59, 57-61.

${ }^{54}$ B. Winter, E. Halford, M. Brouard, Int. J. Mass Spectrom., submitted July 2013.

${ }^{55}$ D. E. Pooley, J. W. L. Lee, et al., manuscript in preparation, 2013.

${ }^{56}$ W. Kockelman, G. Frei, E. H. Lehmann, P. Vontobel, and J. R. Santisteban, Nucl. Instr. Meth. Phys. Res. A., 2007, 578, 421-434.

${ }^{57}$ N. Kardjilov, I. Manke, M. Strobl, A. Hilger, W. Treimer, Th. Krist, M. Meissner, J. Banhart, Nature Physics, 2008, 4, 399-403.

${ }^{58}$ J. R. Santisteban, M. A. Vicente-Alvarez, P. Vizcaino, A. D. Banchik, S. C. Vogel, A. S. Tremsin, J. V. Vallerga, J. B. McPhate, E. Lehmann, and W. Kockelman, J. Nucl. Mat., 2012, 425, 218-227.

${ }^{59}$ M. Strobl, I. Manke, N. Kardjilov, A. Hilger, M. Dawson, and J. Banhart, J. Phys. D: Appl. Phys., 2009, 42, 243001243022.

${ }^{60}$ M. Strobl, Nucl. Instr. Meth. Phys. Res. A, 2009, 604, 646-652.

${ }^{61}$ A. S. Tremsin, J. V. Vallerga, J. B. McPhate, O. H. W. Siegmund, W. B. Feller, L. Crow, and R. G. Cooper, Nucl. Instr. Meth. Phys. Res. A, 2008, 592, 374-384.

${ }^{62}$ A. S. Tremsin, J. V. Vallerga, J. B. McPhate, O. H. W. Seigmund, and R. Raffanti, IEEE Trans. Nucl. Sci., 2013, 60, 578585.

${ }^{63}$ E. S. Wilman, S. H. Gardiner, A. N. Nomerotski, R. Turchetta, M. Brouard, and C. Vallance, Rev. Sci. Instrum, 2012, 83, 013304.

${ }^{64}$ B. Winter, S. J. King, M. Brouard, and C. Vallance, manuscript in preparation, 2013. 